\title{
VGI VISUALISATION TO SUPPORT PARTICIPATORY LAKE MONITORING: THE CASE STUDY OF SIMILE PROJECT
}

\author{
C. A. Biraghi ${ }^{1 *}$, E. Pessina ${ }^{1}$, D. Carrion ${ }^{1}$, M. Brovelli ${ }^{1}$ \\ ${ }^{1}$ Department of Civil and Environmental Engineering, Politecnico di Milano - Lecco Campus, Via Gaetano previati 1/c, 23900 \\ Lecco, Italy - (carloandrea.biraghi, edoardo.pessina, daniela.carrion, maria.brovelli)@ polimi.it
}

Commission IV, WG IV/4

KEY WORDS: VGI visualization, Citizen Science, Lake Monitoring, Usability Evaluation Methods, Participation, Colour theory

\begin{abstract}
:
This study focuses on the role of VGI visualization in encouraging participation to environmental Citizen Science, taking as a case study the mobile application developed within SIMILE (Informative System for the Integrated Monitoring of Insubric Lakes and their Ecosystems) project activities. The main project goal is the protection of water quality for Lugano, Maggiore and Como lakes through a geoinformatics coordination of existing monitoring systems with new data collection methods (satellite images, high frequency sensors and citizen science). This tool enables the producers to perform a community-based monitoring side by side with local authorities and research bodies improving the efficiency of the current protocols.

Referring to ontologies of geospatial data, the VGI samples are analysed under different aspects as their quality, quantity, variety, granularity, spatial and temporal distribution, highlighting their temporary, moving and changing nature. This analysis shows the presence of potential contradictions existing between the application use and the perceived lake water quality, to be mitigated mainly working on the user interface of the map view. So, a study of existing conventions, especially in terms of colours, is compared with the results coming from the application of consolidated survey methodologies to this specific context. Reflections on markers, symbols and attributes of features and maps are presented introducing geovisual analytical solutions for dynamic phenomena. The design solutions adopted are finally presented and validated with a combination of different usability evaluation methods like heuristics and field tests.
\end{abstract}

\section{INTRODUCTION}

\subsection{SIMILE project and its context}

The impact of climate change on our environment is becoming more and more evident. Water is a fundamental and delicate resource in the agenda of the organizations promoting sustainable development (SDGs). In particular, lakes are fragile ecosystems threatened by anthropic pressure and global warming, facing problems as acidification, eutrophication and water-level fluctuation (Vincent, 2009). These issues have to be faced at multiple levels. Efforts in terms of policy and management by the authorities should be supported by people becoming the drivers of a compelling change, minimizing the harmful impact of their activities, adopting virtuous behaviours. Recently, many different Citizen Science projects related to water quality and management have been run across the world, focusing mainly on watersheds and streams (Jollymore et al., 2017).

SIMILE (Informative System for the Integrated Monitoring of Insubric Lakes and their Ecosystems) is a recently started three year project financed by the Interreg Italy-Switzerland 20142021 program (Brovelli et al., 2019). The main project goal is the protection of water quality for Lugano, Maggiore and Como lakes through a geoinformatics coordination of existing monitoring systems with new data collection methods. Images from European Space Agency (ESA) Sentinels are integrated with high frequency sensors, placed on buoys and floating platforms, and with Volunteered Geographic Information (VGI) produced with a Citizen Science approach.

The cross-platform mobile application SIMILE - Lake Monitoring (Pessina et al., 2020) is developed to support the Citizen Science activities, enabling the creation of a network of citizens and offering them several opportunities as:
- contributing to lake monitoring activity by uploading visual observations (presence of foams, algae, drains...) and measurement of water physical properties (temperature, $\mathrm{pH}$, oxygen...)

- having access to the contents of a Business Intelligence (BI) platform, including frequently updated maps and data on lake condition

- being informed on ongoing events and initiatives promoted by the local actors on project themes

- learning sustainable behaviours by answering periodic questionnaires.

This tool ultimately enables the users to perform a communitybased monitoring (CBM) (Conrad \& Hilchey, 2011) side by side with local authorities and research bodies, encouraging contribution (Coleman et al., 2009). This can be seen as a democratization of the environment, an attempt of making environmental science and expertise more accessible to the public, while increasing scientists' awareness of local knowledge and expertise (Carolan, 2006). In respect to most of the citizen science projects dealing with water, SIMILE significantly reduces the commitment required to every volunteer for increasing the chances of involving higher number of participants (Fritz et al., 2017), multiplying the "eyes on the lake". The absence of costs, complicated protocols and experience required, virtually allow everyone having a smartphone eligible for contributing counting on educational opportunity and civic sense as main drivers for motivation.

\subsection{VGI samples inside SIMILE application}

In the following, the mobile application developed in the framework for SIMILE is considered. This study focuses on the initial application view: a map acting as a hub for active citizen participation where observations, measurements and events are

\footnotetext{
* Corresponding author
} 
all displayed. This view allows the user to visualize all contents already inserted by him or others and to insert new ones. As mentioned, there are mainly three kinds of possible contents that can be acquired and visualized with the application: observations, measures and events.

An observation is a picture (the only mandatory input) coupled with the coordinates of the position of the observer and the weather conditions automatically gathered by OpenWeather service, with one or more attributes describing it. The possible attributes that can be acquired together with the picture have been selected exploring the case history of warnings received by the Regional Agency for Environmental Protection (ARPA) of Lombardy and the Environmental department of Ticino canton. They are algae (phytoplankton), foams, oil stains, litters, odours, drains and fauna and each of them contains additional specifications as their intensity, colour, typology and other more specific details. This kind of input is simple as it does not require skills for most of the selectable voices and offers some training via help icons for the most difficult tasks as, for example, recognizing alien species or correctly classifying some natural phenomena.

A measure is the value of a parameter describing a property of lake water. The possible inputs have been identified with the help of the Water Research Institute of National Council of Research (CNR-IRSA) using a balance between simplicity of collection and usefulness for later processing as the main criteria. The input parameters are transparency (Secchi Disk), Temperature, $\mathrm{pH}$, Oxygen and Bacteria, each one with further specific fields mainly regarding the depth at which the measure has been taken and some details of the instrument used.

Events are public meetings of different nature dealing with the project themes. They can be seminars, presentations, beach or water clean-ups, mapathons (measurement campaigns) or even exhibitions and they are uploaded by project partners.

\subsection{VGI ontological analysis}

Examining these three collected elements (Observations, Measures and Events) according to their temporal and spatial dimension, following the principles of the geospatial data ontology by Sun et al. (2019), it's possible to notice that they have peculiar characteristics. The term quality is here intended as the positive/negative dichotomy and not in the sense of precision or reliability as most widely discussed in the literature (Antoniou, 2017). The goal is understanding how something commonly considered as good or bad can affect both the perceived and real water quality of the lake. Table 1 gathers and compares the qualitative, temporal, spatial and thematic characteristics of these elements.

\begin{tabular}{|c|c|c|c|c|}
\hline & Qualitative & Temporal & Spatial & Thematic \\
\hline Observations & negative & $\begin{array}{l}\text { interval } \\
\mathrm{Te} \rightarrow \mathrm{Td}\end{array}$ & $\begin{array}{c}\text { changing } \\
\text { moving }\end{array}$ & changing \\
\hline Measures & $\begin{array}{c}\text { negative/ } \\
\text { positive }\end{array}$ & $\begin{array}{c}\text { Instant } \\
\mathrm{Te} \leftrightarrow \mathrm{Td}\end{array}$ & stable & changing \\
\hline \multirow{2}{*}{ Events } & \multirow{2}{*}{ positive } & interval & \multirow{2}{*}{ stable } & \multirow{2}{*}{ stable } \\
\hline & & $\mathrm{Td} \rightarrow \mathrm{Te}$ & & \\
\hline
\end{tabular}

Table 2. Map elements classification according to geospatial data ontology. $\mathrm{Te}=$ Event Time, $\mathrm{Td}=$ database time.
Observations in most cases correspond to undesired phenomena usually limiting or discouraging lake use, no matter if they're natural or the result of human unproper behaviours. From the temporal perspective they have an event time (Te) " 0 " when they appear and an event time " 1 " when they disappear. Most of them have the maximum degree of heterogeneity as during their life they may vary in size, aspect, colour and position, as they're mainly floating on the water surface. From the spatial point of view, their spatial footprint can change over time and their boundary is vague as different people may understand it differently. The moment of the insertion of an observation represents its database time $(\mathrm{Td})$ and it may happen between these two event times, the extremes of the timeline domain, representing a specific stage of the evolution of the phenomenon. The result is that two observations regarding the same phenomenon, can be different in almost all attributes.

As they are based on lake parameters, measures are neutral values depending only on real water state. They can be considered as a lake sample in a specific position in time and space. Even if the $T e$ and $T d$ correspond, the captured parameter value depends on conditions that change very rapidly, so there's no possibility to know when that measure will no more represent the actual lake condition in that point. Anyway, a high number of measures show a lake under a constant monitoring and can be considered as a positive aspect.

The events can be seen as something positive as they are moments for disseminating project contents in both theory and practice. They can simply provide information or invite citizens to collectively perform actions for lake preservation. The higher the number of participants, the higher is citizens' awareness. They are planned to happen in a precise place and have a specific duration (usually some hours. Nevertheless, before the event starting, they need to be advertised in order to let people know about their existence, and once completed, it can be interesting to share or disseminate their outcomes for a certain time. Differently from the observations, their $T d$ precedes the Te.

From the geometrical point of view, these are all point features. If for measures and events this can be obvious, for observations, even if they represent bidimensional objects (height is almost always irrelevant), the point is still the most convenient representation. In fact, it's almost impossible to draw or define these shapes as polygons on a field mapping activity, especially in this case where it is not possible to walk around the feature, as it's suspended on water, and because of its potential dispersed nature. The missing shape information, for phenomena of considerable dimensions, can be also eventually obtained by the integration with satellite images if needed.

\subsection{Project goals and contradictions}

Once understood the nature of the data the project is expected to deal with through the mobile application, it's time to see how they can help in achieving its goals. As already mentioned, the main project goal is improving lake water quality. In order to achieve that, it's necessary to promote sustainable behaviours among citizens and to improve the efficiency and the coordination of monitoring authorities' activity. A higher water quality, both real or perceived, encourages the use of the lake for recreational purposes and this impacts water quality accordingly to the users' behaviours, but also positively affects the use of the application by bringing more potential users to the lake. The application tries to put together all these aspects by transmitting environmental knowledge, helping authorities 
saving time and resources, returning a trustful picture of the lake and pushing people to take care of the lake by simply observing it. Figure 1 graphically shows these relationships specifying the existence of a perceived and a real quality. Among those goals, the paper focuses on how the map view affects the perceived quality of lake water (represented by the thicker gray arrow) thanks to its symbols, their colour, quantity and relationship with the background.

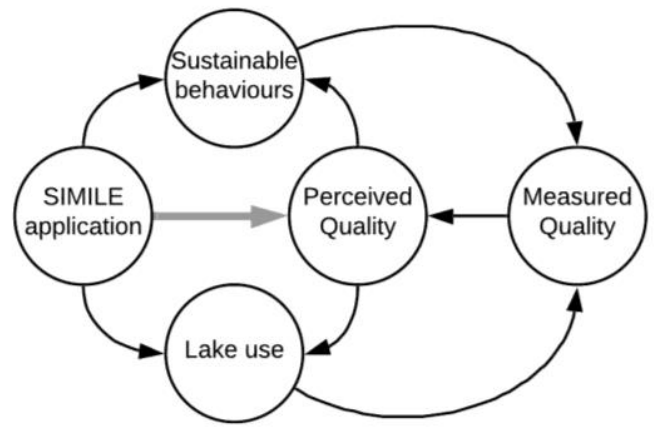

Figure 1. Scheme on how SIMILE application interacts with project goals promoting sustainable behaviours, encouraging

lake use and increasing perceived lake water quality

The quality of the features is particularly relevant for the selected case and its representation criteria may strongly affect citizen involvement. The challenge is overcoming the paradox of encouraging lake use while displaying mainly negative contents (observations), even in case of good lake water conditions.

\subsection{Previous studies on geovisualisation}

An exploration of geo-visualisation, visuospatial displays and perception studies helped defining the selected approach. Deparday (2010), in his work, investigates the potential of several interactive geo-visualization techniques including filtering, dynamic spatial aggregation developing a prototype. Brychtova \& Coltekin (2016) combine eye-tracking with traditional usability metrics to assess the effectiveness of visualisation and Nivala et al., (2003) explore field tests to identify, at an early stage of the project, preliminary design principles for maps in small displays. Klettner (2019) investigates shape proximities and cognitive relation in symbology, while Stone (2006) defines some guidelines for choosing colours. The main challenge of SIMILE app map view, using Chandler's (2002) words, "is changing the form of the signifier while keeping the same signified in order to generate different connotations". Changes in visual map style influence the map readers' judgments, trust, liking and recall (Muehlenhaus, 2013), but there still remains the need for a differentiated perspective in terms of the "identification and articulation of the basic visual variables that can be manipulated to encode information" (Maceachren et al., 2012). For doing that, existing graphical conventions used in the water monitoring field have been studied, discovering the predominance of colour as the feature in charge to represent quality. In fact, there is a limited set of simple symbols (dots, polygons, flags), changing from website to website, used as constants while colours change.

\subsection{Colour meaning in water context}

To analyse symbols used to represent water quality, given the local, but scalable, nature of the initiative, the attention has been focused on the products of the regional agencies and the environmental associations or bodies operating in the same context (ARPA Lombardy, ARPA Piedmont, Ticino Canton, Legambiente). The "Bandiera Blu" (blue flag) certification assigned by the Foundation for Environmental Education (FEE) to the highest quality swimming sites has also been included. Figure 2 collects the colours used for different thematic representations related to water quality by the mentioned authorities. The result is an almost total accordance among them as they all follow the guideline of the international Water Framework Directive (EC WG 3.1 - GIS, 2003). Section 1.4.2 indicates the colours to represent the ecological status and section 1.4.3 describes the chemical status conventions.

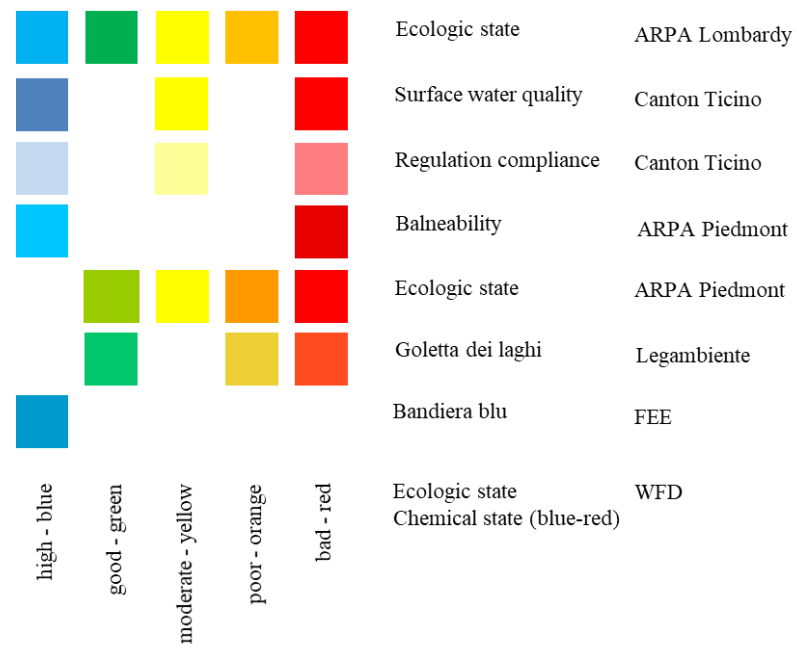

Figure 2. Collection of the colours used by the local authorities in web services about different aspects of water quality.

The colours used by the water portal of the Italian Minister of Health, not reported in Figure 2, follow this scale with few modifications. Blue indicates a measurement point, without quality implications, green is used for swimming waters, orange for forbidden waters with no specification of the cause, red for polluted waters and purple is specific for algal blooms.

In order to contextualise these outcomes in the broader colour theory, the literature of colour meaning, which is mainly coming from different disciplinary sectors as marketing, has been investigated. Palmer \& Schloss (2010) suggest that people like colours associated to the objects they like and prove it through an empirical test. Won \& Westland (2017) tested the colour meaning by asking people to set a value on a slider having as extremes two opposite words (e.g. warm-cold) to express their feeling about the observed colour patch. The colours analysed are fixed while words' couples changed from author to author. Then, the same test is repeated on colours applied to some objects (e.g. packaging). It emerged a difference between the meaning of a colour in absolute terms and when applied to a specific context. Considering that the environmental field has not been studied yet from this perspective, an attempt has been made to adapt the consolidated techniques to a new discipline. The approach used by Won \& Westland (2017) has been replicated adapting it to the new context both in terms of topic and visualization support. Two surveys have been created using the Socrative application and answered directly from mobile devices, the final user interface of our application. The tests have been conducted on a sample of 18 users ranging from 24 to 91 years old with an average of 30 , composed by $55 \%$ of females and $45 \%$ of males. 


\section{COLOUR MEANING AND USABILITY TEST}

\subsection{Test 1: absolute colour meaning}

In test 1 , users were asked to select a number between 0 and 10 to express the meaning of each colour for every couple of words. Values lower than 5 refer to the first and left term of the couple, while values higher than 5 refer to the second and right one. The closer is a value to one of the extremes, the stronger is the correspondence, while values between 4 and 6 can be seen as uncertain. New couples of words have been substituted to the ones used by Won \& Westland (2017) (masculinelfeminine, warm/cold, expensivelinexpensive, modern/traditional, elegant/vulgar) to explore new absolute colour meanings for the same six colours (beige, black, blue, green, red, yellow) presented separately. These couples have been selected by thinking at possible criteria to customize visualization and are the following:

- Positive/negative, to distinguish aspects related to environmental quality

- Past/future, to emphasize the temporal dimension of the possible map symbols

- Amateur/professional, to highlight the level of expertise of the users and their reliability

- Regular/exceptional, to differentiate periodic and sporadic activities

- Mine/yours, to evaluate the possibility of filtering between user's own contents and the others.

Before starting the test, the possible overlapping between these new couples and the previously tested word-colour correlation (Won \& Westland, 2017) has been verified by assigning to each word a numerical value.. A value of "2" was given to those words with a strong match with one of the extremes of the new couples, while "1" for weaker or more arguable correspondences. In case of no evidence, no value was assigned. For example, the word "death" is universally considered a negative (value $=2$ ) event, can be seen as something exceptional (value $=1$ ) while has no link with words like amateur or professional. Considering that these words already had correspondences with one or more colours, this allowed to see preliminary correspondences between the same colours and the new couples. Mean values over a maximum score of 2 and match count for colour, among the results coming fro Won \& Westland (2017), are reported in brackets in the following. The most evident matches were Negative words with black (mean over maximum of 2 , match count: 1.8/2, 6), Positive with green $(1.8 / 2,9)$ and blue $(2 / 2,4)$, and Regular with green $(1.3 / 2,7)$. No link has been found for yellow, and for the Mine/Yours couple.

After explaining to the users how to perform the test, before starting, two examples have been provided using words (slowlfast, begin/end) and colours (fuchsia) as a training set. Table 2 presents the synthetic results of the new test in terms of mean values computed on users' answers. As indicated in the legend, blue indicates a high correspondence with the right (second) word, red with the left (first) one and white or lighter nuances of red and blue the uncertain values.

The results confirmed the main existing correlations in terms of absolute colour meanings and their intensity with extreme mean values (Black-Negative 2.5, Blue-Positive 8.00, Green-Positive 9.11 and Green-Regular 3.44).

\begin{tabular}{|c|c|c|c|c|c|}
\hline & 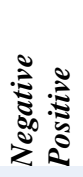 & $\underset{0}{0}$ & 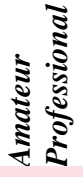 & 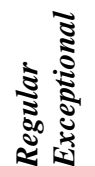 & $\stackrel{\Xi}{\Xi}$ \\
\hline Beige & 5.56 & 4.89 & 4.11 & 3.28 & 4.89 \\
\hline Black & 2.50 & 3.11 & 6.72 & 5.00 & 6.33 \\
\hline Blue & 8.00 & 7.78 & 6.44 & 3.33 & 5.67 \\
\hline Green & 9.11 & 6.78 & 4.56 & 3.44 & 4.56 \\
\hline Red & 4.5 & 4.83 & 5.06 & 8.56 & 5.11 \\
\hline Yellow & 5.06 & 5.28 & 4.33 & 6.00 & 5.94 \\
\hline Legend & 0.00 & 2.50 & 5.00 & 7.50 & 10.00 \\
\hline
\end{tabular}

Table 2. Test 1 results, mean of the 18 answers

Beige had an association with the word regular. Black resulted in tending towards past more than future but still with people on both extremes. It also had minor match with professional and yours. Blue had other three lighter associations with the word future, professional and regular but still with a high range. The most impressive result is the match between red and exceptional as no one assigned it a value lower than 7. Yellow-yours minimal match doesn't represent something particularly significant. This test allowed to investigate the association of these colours to terms not yet included in the previous studies.

\subsection{Test 2: colour meaning in the environmental contex}

At a second stage, the users have been asked to indicate one or more colours, among the six already used, that better represent a word or short sentence contained in every question. In this case the terms used were more explicitly related to the project context. This way of assessing the difference between the absolute colour meaning and the contextual one is different from the one used by Won \& Westland (2017), where colours were applied to different products packaging.

A set of 6 words (sand, tyre, sky, grass, fire, lemon) have been used as a test for the comprehension of the survey as their association to one colour is almost universally clear. The other words are divided into sections to facilitate the reading of the results. The first and the second sections contain the observations and measure tags insertable within the mobile application, third and fourth include negative and positive environmental terms, the fifth includes words indicating trust and finally the last section shows other terms related to the criteria for customizing visualisation. Table 3 contains all words included in the test in the rows and the preferences attributed to each colour in the corresponding column. The test was performed by the same 18 users of test 1 . The possibility of expressing more than one option (up to 6) allowed not only to understand what colour was more commonly associated to a concept, but also how exclusive was this association and if some of the remaining colours also have a significant match. A set of simple metrics have been used to assess the results:

C1/Pmin,

Pmin/Ptot,

$C 2 / C 1$ 
Where $\quad \mathrm{C} 1=$ total preferences of the $1^{\text {st }}$ ranked colour Pmin = number of participants $(18)$

Ptot $=$ total preferences indicated $\mathrm{C} 2=$ total preferences of the $2^{\text {nd }}$ ranked colour

\begin{tabular}{|c|c|c|c|c|c|c|c|c|}
\hline & Words & 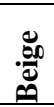 & 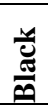 & $\stackrel{\mathscr{g}}{ٍ}$ & 递 & تֶ. & 产 & 胥 \\
\hline \multirow{6}{*}{ 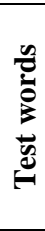 } & Sand & 18 & 2 & & & 3 & 5 & 28 \\
\hline & Tyre & 2 & 16 & & & 2 & & 22 \\
\hline & Sky & & 2 & 18 & & 3 & 2 & 25 \\
\hline & Grass & 1 & & & 18 & & 1 & 20 \\
\hline & Fire & & 3 & 2 & & 18 & 7 & 30 \\
\hline & Lemon & 1 & & & 3 & & 18 & 22 \\
\hline \multirow{7}{*}{ 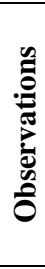 } & Algae & 2 & 1 & 1 & 17 & 2 & 3 & 26 \\
\hline & Foams & 13 & 2 & 7 & 1 & & 4 & 27 \\
\hline & Fuel & 2 & 10 & 3 & 4 & 5 & 5 & 29 \\
\hline & Waste & 4 & 14 & 1 & 2 & 1 & 1 & 23 \\
\hline & Odours & 8 & 3 & & 5 & 2 & 7 & 25 \\
\hline & Drains & 4 & 12 & 7 & & 2 & 2 & 27 \\
\hline & Fauna & 5 & 3 & 2 & 11 & 5 & 5 & 31 \\
\hline \multirow{5}{*}{ 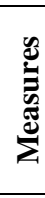 } & Transparency & 5 & 1 & 12 & & & 2 & 20 \\
\hline & Temperature & 1 & & 7 & 2 & 13 & 3 & 26 \\
\hline & $\mathrm{pH}$ & 3 & 2 & 9 & 5 & 3 & 9 & 26 \\
\hline & Oxygen & 2 & & 14 & 3 & & & 19 \\
\hline & Bacteria & 7 & 12 & 1 & 3 & 4 & 2 & 29 \\
\hline \multirow{5}{*}{ 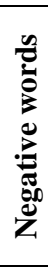 } & $\begin{array}{l}\text { Low } \\
\text { environmental } \\
\text { quality }\end{array}$ & 4 & 13 & & & 9 & 4 & 30 \\
\hline & Problem & & 7 & 1 & & 14 & 4 & 26 \\
\hline & Warning & & & 1 & & 15 & 6 & 22 \\
\hline & Pollution & 4 & 17 & & & 4 & 4 & 29 \\
\hline & Alien species & 4 & 3 & 5 & 6 & 3 & 5 & 26 \\
\hline \multirow{5}{*}{$\begin{array}{l}0 \\
0 \\
0 \\
0 \\
0 \\
0 \\
0 \\
0 \\
0\end{array}$} & $\begin{array}{l}\text { High } \\
\text { environmental } \\
\text { quality }\end{array}$ & 1 & & 12 & 15 & & 1 & 29 \\
\hline & Safety & 3 & & 11 & 10 & 1 & & 25 \\
\hline & Clean & 3 & & 14 & 9 & & 3 & 29 \\
\hline & User friendly & 3 & 1 & 10 & 7 & 3 & 4 & 28 \\
\hline & Ecosystem & 1 & & 6 & 17 & & 2 & 26 \\
\hline \multirow{5}{*}{$\begin{array}{l}0 \\
0 \\
0 \\
0\end{array}$} & Reliable & 3 & & 9 & 13 & 1 & 1 & 27 \\
\hline & Scientific & 1 & 2 & 11 & 8 & 3 & & 25 \\
\hline & Official & 1 & 7 & 11 & 2 & 4 & & 25 \\
\hline & Efficient & 1 & & 6 & 12 & 2 & 5 & 26 \\
\hline & Learning & 2 & & 12 & 7 & 6 & 3 & 30 \\
\hline \multirow{7}{*}{ 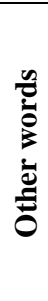 } & Open source & 2 & 1 & 10 & 13 & & 1 & 27 \\
\hline & Mobile app & 2 & 3 & 12 & 6 & 1 & 3 & 27 \\
\hline & $\begin{array}{l}\text { Monitoring } \\
\text { activity }\end{array}$ & 4 & 1 & 8 & 4 & 5 & 6 & 28 \\
\hline & Widespread & 5 & 1 & 6 & 4 & 2 & 6 & 24 \\
\hline & Personal & 6 & 3 & 4 & 5 & 5 & 1 & 24 \\
\hline & Event & 1 & 1 & 9 & 6 & 7 & 3 & 27 \\
\hline & Participation & 6 & & 6 & 6 & 5 & 3 & 26 \\
\hline
\end{tabular}

Table 3. Test 2 results, sum of all the preferences per word per colour and total number of preferences of the word

For $C 1 / P \min$ high values mean strong associations between word and colours. For Pmin/Ptot, high values mean a question with few alternatives per answer where participants mainly expressed only one preference. For $C 2 / C 1$, low values mean predominance of one colour over the others and a non-dualistic majority.As expected, the values of $C 1 / P \min$ for the test words is equal or close to 1 , the Pmin/Ptot is quite high and $C 2 / C 1$ is very low.

The results showed that most of lake phenomena, contained in the observations section, are not immediately associated with one specific colour. The only exceptions are algae (94\% green) and litters (78\% black). Moreover, other two of them (fuel and drains) have black as the first ranked colour, even if with low values, confirming its negative perception. Odours, not immediately related to sight, have the lowest values and have been mainly associated to beige and yellow. The situation is similar with measures with the oxygen, which has been associated to blue as most meaningful match (78\%) and blue as the overall most frequent colour with a possible link to its professional (6.44/10) meaning emerging from test 1 . The most uncertain is the case of $p H$ with a low C1/Ptot, the lowest $P m i n / P t o t$ and a $C 2 / C 1$ value equal to 1 . This might be due to the fact that the $\mathrm{pH}$ colour ramp is well known also by nonexperts and includes four of this colours, two on the acid side (red for $p H=0$ and yellow, $p H=3$ ) one in the neutral centre (green, $p H=7$ ) and one for the alkaline part (blue, $p H=11$ ). Temperature has highest values in red and blue, normally associated to warm and cold. Bacteria is associated to black $(68 \%)$ confirming the negative meaning of this measure and the relatively high rate of beige (39\%) suggests that probably a mix of the two colours (brown) could be the most suitable one.

The negative words section contains the most interesting results. In fact, if the preferences for black have already been explained, the association with red is something new that can be considered as related to this specific context. Red has many positive meanings but when it comes to the environmental field the situation changes. Words as problem $(78 \%)$ and warning (83\%), associated with red, are correctly considered exceptional situations (red in test 1) respect to a normality made of a good environmental quality. Their negative meaning is confirmed by a correspondence with a low environmental quality (50\%). This result, achieved by surveying also non expert users, is in accordance with the Water Framework Directive colour ramp associating red to bad quality (EC WG 3.1-GIS, 2003). Positive words totally confirmed the dominance of green and blue with the first one leading when the environmental meaning is more explicitly indicated (high env. quality, $83 \%$, ecosystem, 94\%). Clean (78\%) and safety (61\%), the most suitable characteristics of water bodies for recreational uses, see blue as the leading colour, as expected. That is also because blue is associated to water in its pure and natural state.

Trust words show the same trend with a bit more of blue and the presence of black as second term for the word official. These words correspond to desired image for the citizen science application and service under development as they also have a commonly accepted positive meaning. The same can be said for the word open source in the last section. The remaining terms showed no evident connection with one colour. Personal and participation remained highly uncertain as visible from the results of the mine-yours couple in test 1 . Event doesn't have a clear result but the colours with more preferences are blue, red and green $(9,7,6$ preferences).

\subsection{Definition of the application symbols}

The results of both tests gave useful insights for defining the symbology of the elements of the mobile application. The map view, in the ideal scenario, should present a good number of symbols and communicate a high quality monitored environment. Given the high percentage of negative meanings 
inside the app contents (observations), it has been decided to keep the representation as neutral and uniform as possible both in terms of symbols and colours. A single kind of "pin-like" marker with a circular space in the head of the pin has been combined with inner symbols.

No specific colour or symbol has been dedicated to each observation or measure also because of the possibility given to the user of inserting more than one parameter inside the same observation. In addition, a legend with too many different labels could have a negative impact on the map readability. The only distinction has been made between observations and events, as they are separate features with different paths inside the application workflow, varying colour and inner symbol within the same marker shape to distinguish them. Marker size used is $465 \times 850$ pixels for retina display devices (SVG Marker, www.Ya-webdesign.com). Figure 3 shows all different symbols that it is possible to find on the application map view.
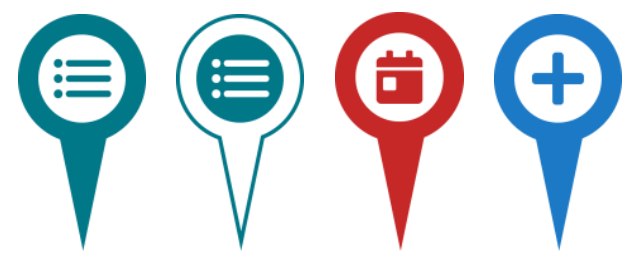

Figure 3. Application symbols. From left to right: observation, my observation, event, manually selected position

A single colour has been chosen for each item using white as a neutral background to increase contrast and visibility. For observations and measures, the SIMILE project colour, a combination of blue and green (RGB 0, 120, 136), has been used in order to communicate a good environmental quality granted by a regular monitoring activity and efficient authorities, as emerged from the previous tests. This colour, taken from the corresponding axes (5) of the Interreg program, makes also a good contrast with the light blue of lake water of the OpenStreetMap (RGB 171, 211, 223) background tiles. The choice of the symbol has been driven by the need of adopting something quite generic and able to communicate the possibility of inserting more than one option. For that reason, a "list" icon has been chosen (list-filled, www.ionicons.com). A negative version of this marker has been used to recognize user's own observations (second symbol in Figure 3).

The events have been associated to red (RGB 198, 40, 40) for their exceptional nature and because their possible location on any point of the map, usually outside water, they required a colour able to contrast with many others (buildings, grey RGB 218, 208, 199; blocks, light brown, 244, 241, 234; streets, white, 252, 251, 247; squares, grey-purple, 221, 220, 234; parks, light green, 207, 246, 211). The symbol used is a stylised calendar (calendar day, www.fontawesome.com), commonly associated to scheduled events.

The last symbol is a temporary and operative one as it allows users to manually select a position different from the one indicated by their GPS for the insertion of the observation. The blue colour (RGB 28, 121, 198) comes from the GPS circle, taken from Google Maps as considered the most popular map service, and use the "+" as inner symbol (+, www.material.io), the same used for inserting a new observation. In this way, no item with a colour inspiring a negative meaning will ever appear inside the lake, as the events are located on the land, and this without hiding real water conditions. Observation content doesn't affect its representation but is visible by opening it.

\subsection{Data quantity and map balance}

Another key element for map balance (Haklay, 2010) is the total amount of visible data. Considering that the quantity of input data will be strongly dependent by the diffusion of the app and by the occurrence of phenomena, there was the need of understanding the volumes and the trends of those phenomena related to the observations in order to predict potential uneven distribution of volunteer contributions.

It's well known in limnological studies (Bertoni, 2018) that these natural phenomena have a higher frequency from late spring to the end of summer. Confronting with the local authorities, it emerged that in the peak moments it's hard to receive more than one warning per day per lake. These low numbers are on one side a proof of the good condition of the lakes under study in SIMILE project, but it can also be explained by the current warning procedure, quite demanding and inefficient especially on the Italian side. In fact, in order to officially send an alert, citizens must call a telephone number and wait on the observation site for the arrival of an officer, similarly to what happens with the law enforcements. The Swiss procedure is similar to the one implemented with the mobile application as it allows to send a picture, but it's based on a website and the information is all in form of free text, with no tags, coordinates or weather conditions attached. The introduction of the application may tremendously reduce the waiting time for the Italian side from up to $30 \mathrm{~min}$ to $1-2 \mathrm{~min}$ with the advantage of giving to the authorities preliminary information for evaluating the phenomenon before going in the field. The positive aspects of the swiss procedure will be combined with the benefits of a tool developed explicitly for that saving most of the time required for digitizing the texts.

The number of contributions to a volunteer project is one of the factors that give the perception of how much a service is currently used. The feeling that the service you are contributing to is alive is an important aspect for motivation. On the other hand, an overwhelming amount of contents may risk generating a feeling of unnecessity, ultimately discouraging contribution. For this reason, in order to limit these risks, the concepts of "expiring time" and "map limit density" have been introduced. The expiring time as an attribute of the VGI adds a degree of flexibility to the developers, that by extending or compressing it, and linking it to a gradually vanishing visualization strategy, can ensure a constant good readability while introducing the possibility to distinguish between older and newer observations. This can be particularly helpful in case of overlapping observations describing a changing phenomenon. The expiring time can be generic or tailored on the content of an observation according to the average duration of the physical phenomenon they represent.

The map limit density is simply the maximum number of features that can be contained in a certain spatial unit. The most suitable spatial unit has been identified in the OSM tile at the maximum zoom level allowed inside the application (level 18). The choice of using only the maximum zoom level as a "filtering" criterium is due to the use of Leaflet MarkerCluster (https://github.com/Leaflet/Leaflet.markercluster) algorithm to group together features of the same kind at a mutual distance lower than a threshold one, in order to display the markers only when they can be clearly visible. According to the equation (1) available on the Wikipedia page of OSM, a tile at a latitude of 
$46^{\circ}$, an average of project lakes' coordinates, has nearly $108 \mathrm{~m}$ side.

$$
\text { Tile side }=C \cdot \cos \left(\text { latitude) } / 2^{\wedge}\right. \text { zoomlevel }
$$

where $\quad \mathrm{C}$ is the equatorial circumference of the Earth

Considering that level 18 correspond to a 1:2000 scale, a tile at that zoom level will occupy nearly $5.4 \mathrm{~cm}$, almost corresponding to the shortest side of the screen of a mobile device. Both expiring time and map limit density values could be defined a priori in the mobile application, but their value and usefulness strongly depend by the number of features uploaded in the application with a certain frequency. For this reason, these parameters will be tuned after at least one year of application life and use. After explaining the reasons behind the visual aspects of SIMILE application map view, a usability test has been performed. A beta version has been developed and sent to 18 users selected among the project partners, together with a test prepared using Google Forms.

The usability test has been constructed referring to different Usability Evaluation Methods (UEM). It is divided in three sections:

- Tasks: four guided activities covering most of the application functionalities. A synthetic description of the task goal is provided and followed by detailed step by step instructions

- Questions: 24 sentences where the user is asked to express the level of agreement on a scale from 1 to 5

- Conclusions, additional space for free comments divided in four sections (visualisation and recognizability, learnability and memorability, efficiency and other).

Considering that the possibility of a real on field test depends by the presence of one or more undesired phenomena, those situation has been simulated by attaching to the test the information to be included in the different observation in form of picture (task 1) or text (task 2) to be interpreted. In order to better evaluate the memorability of the app flow, the same step, no matter its complexity, was explained only once in detail. The work of Kuparinen et al. (2013) has been used as a guide for choosing the most appropriate sentences to be used. As its concept of MMA deals mainly with routing services, it has been integrated with the classical principles for systems of Nielsen (1993) and the work of Nivala et al. (2003) on field tests to better cover the application functionalities. The complete list of sentences, available in the usability test, has been divided by the corresponding aspect of usability heuristics they refer to:

Visibility of system status

Match between the system and the real world

User control and freedom

Consistency and standards

Error prevention

Recognition rather than recall

Flexibility and efficiency of use

Aesthetic and minimalist design

Balanced and simplistic visual design

\subsection{Results}

Usability assessment results have been positive and are here reported and commented. All the users completed the tasks in a reasonable time. Only a minor bug that caused the disappearance of an event feature caused some problems in the fourth task. All activities have been performed in an average time of 2-4 minutes from people at the very first experience with the app and no preliminary introduction. Among those, we had someone taking only 10-30 second to perform them. This is an encouraging sign of a sensible reduction of the needed time as long as users become more familiar with the application.

\begin{tabular}{c|cccc} 
& Task 1 & Task 2 & Task 3 & Task 4 \\
\hline Success rate & 1.00 & 1.00 & 1.00 & 0.89 \\
Average (sec) & 146 & 207 & 150 & 136 \\
Minimum (sec) & 10 & 30 & 20 & 30
\end{tabular}

Table 5. Usability test task results

Most of the sentences had a positive feedback with no evaluation below 3 on a scale from 1 to 5 . Only the $13 \%$ of the users (question, mark: 10, n.d.) encountered a problem but had no difficulty in understanding $(11,4.5 / 5)$ and solving it (12, $5 / 5)$. Most interesting results are those related to symbols $(9$, $4.56 / 5)$, colours $(23,4.35 / 5)$ and representation at the different scales $(17,4.39 / 5)$ together with the comments of the visualisation section:

"Symbols are clear and colour palette well balanced, not annoying for the eye, granting a good usability."

Even the flow and the hierarchic structure of the contents in the process of inserting an observation has been appreciated (7, $4.56 / 5 ; 13,4.28 / 5 ; 19,4.44 / 5)$ and no particular need of a more diversified or granular representation emerged $(22,3 / 5)$ :

"The observation process is very linear and doesn't need of further instructions. Following the app flow, it's possible to send the observation in few steps."

Even if only time will tell more about the real efficiency of this app and the potential of using its data to validate the other project products, another positive comment suggests that the potential trade-off existing between data flow and accuracy (Connors et al., 2012) could have been overcome:

"The time/detail ratio is good, in few seconds is possible to insert the main data with a high level of detail."

\section{CONCLUSIONS}

This study focuses on the role of VGI visualization in encouraging participation to environmental Citizen Science. The ontological investigation of the VGI allowed to demonstrate their peculiar nature of temporary, moving and changing features and the need for specific strategies for their visualization. In addition, a different meaning of data quality, intended as positive and negative features, is considered demonstrating the importance of mitigating its visual impact for the perceived environmental quality. Colour theory techniques have been used to explore visualization criteria of interest for the selected case study and verifying colour meaning in the environmental monitoring field. The main outcome is the negative acceptation of the red, in accordance with the WFD but not evident from the sole absolute colour meaning investigation. The concepts of expiring time and map limit density are introduced as a possible solution for the visuospatial designer to control data quantity and assure a balanced map view, with the goal of fostering participation. A design strategy for the map view is presented and assessed with different UEM. The positive results of the test are a convincing proof of the success of the implemented visualization strategy. The next steps of the research will see the monitoring of citizen volunteer contribution over the project duration to verify if the appreciation of the user interface, appropriately supported by a 
communication strategy, will translate into a high and continuative participation rate.

\section{ACKNOWLEDGEMENTS}

The research described in this paper is part of SIMILE (Informative System for the Integrated Monitoring of Insubric Lakes and their Ecosystems) project and has been funded with support from the European Commission within the Interreg Italy-Switzerland 2014-2021 program.

\section{REFERENCES}

Antoniou, V., 2017: Assessing VGI Data Quality. In Mapping and the Citizen Sensor. doi.org/10.5334/bbf.g

Bertoni, R., 2018: Laghi e Scienza, introduzione alla limnologia. Aracne, Rome, Italy.

Brovelli, M.A., Cannata, M., \& Rogora, M., 2019: SIMILE, a geospatial enabler of the monitoring of Sustainable Development Goal 6 (ensure availability and sustainability of water for all). Int. Arch. Photogramm. Remote Sens. Spatial Inf. Sci., XLII-4/W20(4/W20), 3-10. doi.org/10.5194/isprsarchives-XLII-4-W20-3-2019

Brychtova, A., \& Coltekin, A., 2016: An Empirical User Study for Measuring the Influence of Colour Distance and Font Size in Map Reading Using Eye Tracking. Cartographic Journal 53(3), 202-212. doi.org/10.1179/1743277414Y.0000000103

Carolan, M.S. 2006: Science, expertise, and the democratization of the decision-making process. Society and Natural Resources 19(7), 661-668. doi.org/10.1080/08941920600742443

Chandler, D. 2002: Semiotics the Basics. Routledge, Abingdon.

Coleman, D.J., Georgiadou, Y., \& Labonte, J. 2009: Volunteered Geographic Information: The Nature and Motivation of Produsers. International Journal of Spatial Data Infrastructures Research 4, 332-358. doi.org/10.2902/17250463.2009.04.art16

Connors, J.P., Lei, S., \& Kelly, M. 2012: Citizen Science in the Age of Neogeography: Utilizing Volunteered Geographic Information for Environmental Monitoring. Annals of the Association of American Geographers. doi.org/10.1080/00045608.2011.627058

Conrad, C.C., \& Hilchey, K.G. 2011: A review of citizen science and community-based environmental monitoring: Issues and opportunities. Environmental Monitoring and Assessment. doi.org/10.1007/s10661-010-1582-5

Deparday, V. 2010. Enhancing Volunteered Geographical Information (VGI) Visualization with Open Source Web-Based Software. Master thesis in Geography, University of Waterloo, Ontario, Canada.

European Commission Working Group 3 - GIS, 2003. Common Implementation Strategy for the Water Framework Directive (2000/60/EC) Implementing the Geographical Information System Elements (GIS) of the Water Framework Directive. europa.eu.int
Fritz, S., See, L., \& Brovelli, M.A. 2017: Motivating and Sustaining Participation in VGI. In Mapping and the Citizen Sensor. doi.org/10.5334/bbf.e

Haklay, M. 2010: Interacting with geospatial technologies. John Wiley, Hoboken, New Jersey, USA.

Jollymore, A., Haines, M.J., Satterfield, T., \& Johnson, M.S. 2017: Citizen science for water quality monitoring: Data implications of citizen perspectives. Journal of Environmental Management 200(June), 456-467. doi.org/10.1016/j.jenvman.2017.05.083

Klettner, S. 2019: Why Shape Matters-On the Inherent Qualities of Geometric Shapes for Cartographic Representations. ISPRS International Journal of Geo-Information 8(5), 217. doi.org/10.3390/ijgi8050217

Kuparinen, L., Silvennoinen, J., \& Isomäki, H. 2013. Introducing Usability Heuristics for Mobile Map Applications. Proceedings of the 26th International Cartographic Conference. Dresden, Germany

Maceachren, A.M., Roth, R.E., O’Brien, J., Li, B., Swingley, D., \& Gahegan, M. 2012: Visual semiotics \& uncertainty visualization: An empirical study. IEEE Transactions on Visualization and Computer Graphics 18(12), 2496-2505. doi.org/10.1109/TVCG.2012.279

Muehlenhaus, I. 2013: The design and composition of persuasive maps. Cartography and Geographic Information Science 40(5), 401-414. doi.org/10.1080/15230406.2013.783450

Nielsen, J., 1993: Usability engineering. Academic Press, Cambridge, Massachusetts, USA.

Nivala, A., Sarjakoski, L.T., Jakobsson, A., \& Kaasinen, E. 2003. Usability evaluation of topographic maps in mobile devices. Proceedings of the 21st International Cartographic Conference (ICC). Durban, South Africa.

Palmer, S.E., \& Schloss, K.B. 2010: An ecological valence theory of human color preference. Proceedings of the National Academy of Sciences of the United States of America 107(19), 8877-8882. doi.org/10.1073/pnas.0906172107

Pessina, E., Carrion, D., Biraghi, C.A., \& Brovelli, M.A. 2020. Crowdsourcing water quality with the SIMILE app. Int. Arch. Photogramm. Remote Sens. Spatial Inf. Sci.

Stone, M. 2006: Choosing Colors for Data Visualization. Perceptual Edge. doi.org/10.1111/j.1600-0854.2008.00863.x

Sun, K., Zhu, Y., Pan, P., Hou, Z., Wang, D., Li, W., \& Song, J. 2019: Geospatial data ontology: the semantic foundation of geospatial data integration and sharing. Big Earth Data. doi.org/10.1080/20964471.2019.1661662

Vincent, W.F. 2009: Effects of Climate Change on Lakes. In Encyclopedia of Inland Waters. Academic Press, Cambridge, Massachusetts, USA. doi.org/10.1016/B978-0123706263.00233-7

Won, S., \& Westland, S. 2017: Colour Meaning and Context. Color Research and Application 42(4), 450-459. doi.org/10.1002/col.22095 